\title{
Age at hormonal onset of puberty based on luteinizing hormone, inhibin B, and body composition in preadolescent US girls
}

\author{
O. Yaw Addo', Bradley S. Miller², Peter A. Lee 3 , Mary L. Hediger ${ }^{4}$ and John H. Himes
}

BACKGROUND: Hormonal indicators could be useful for detecting early pubertal onset, but there is little research on how they are related to puberty in US girls. We determined median age at hormonal onset of puberty based on luteinizing hormone $(\mathrm{LH})$ and inhibin $\mathrm{B}(\mathrm{InB})$ and explored the extent to which body composition moderates this timing process.

METHODS: We analyzed anthropometric and hormone data of 698 US peri-pubertal girls ages 6-11.99 y who had participated in the Third National Health and Nutrition Examination Survey (NHANES III), 1988-1994.

RESULTS: Median age of hormonal onset of puberty was 10.43 y by LH and 10.08 y by InB cut-offs (1.04 mIU/ml for LH and 17.89 $\mathrm{pg} / \mathrm{ml}$ for InB). Postnatal weight gain modulated onset, making it earlier by 10-11 mo among the highest (greater than +1 SD) relative to normal weight gainers. Onset occurred first in nonHispanic black (NHB) girls, 10.08 y (95\% confidence interval (Cl): 10.07-10.09), followed by Mexican-American (MXAM) at 10.64 y (95\% Cl: $10.63-10.65)$, and at 10.66 y (95\% Cl: 10.66-10.67) for non-Hispanic white (NHW) girls using LH. With $\ln B$, onset occurred first in MXAM girls at $9.9 \mathrm{y}$, and at $10.3 \mathrm{y}$ and $10.4 \mathrm{y}$ for their NHB and NHW peers, respectively.

CONCLUSION: Preadolescent weight gain lowers the age at hormonal onset as defined by LH concentrations. Preventing obesity in childhood may also avert the earlier initiation of the maturation process even at the hormonal level.

A lthough breast bud palpation has been recommended for assessing puberty in epidemiologic studies $(1,2)$, it is often not feasible in nonclinical settings due to privacy concerns and can be inaccurate in overweight girls. As a result, menarche is frequently used as the only marker of puberty. Although the timing of puberty is influenced by several factors including genetics, nutrition, adiposity, ethnicity, and environmental pollutants $(3,4)$ as well as size at birth $(5,6)$, it also has a strong and obligatory endocrine component. Puberty is triggered by reactivation of the hypothalamic-pituitary-gonadal axis which stimulates the release of luteinizing hormone (LH) and follicle-stimulating hormone (FSH) from the pituitary gland, which in turn induce gonadal maturation (7), including inhibin $B(\operatorname{InB})$ secretion by the ovaries. The elevation of LH circulating levels during puberty are of much greater magnitude than the rise of FSH. The changes of InB, an index of ovarian response to gonadotropin, particularly FSH stimulation, have not been well studied. Hence, the changes of both of these hormones can be markers of onset of pubertal hormonal secretion.

While hormonal indicators of onset of puberty would be useful for early detection as recommended by an expert panel in $2008(1,2)$, there is little research on how pubertal hormones are related with pubertal timing in US children. The availability of nationally representative hormone data ( $\mathrm{LH}, \mathrm{InB})$, although not a comprehensive set of indicators, for a peri-pubertal sample of US girls provides an opportunity to elucidate some of the auxological factors that influence this process in US children. Because birthweight, preadolescent weight and puberty are sequentially interrelated, birthweight has the potential to mediate postnatal weight gain and puberty via various adaptive mechanisms in concert with environmental influences. We examined the extent to which these factors potentially influence the timing of pubertal onset in girls and assessed the relative importance of prenatal and postnatal growth measures in predicting two hormones in peri-pubertal US girls.

Even though studies have shown a downward trend in the ages of physical onset of puberty worldwide $(2,8)$, this trend is more pronounced for initial onset events and less so for age at menarche in girls, a relatively late event and occurring only after substantial hormone stimulation. We estimate the age at hormonal onset of puberty and explore the extent to which weight gain affects the timing. Understanding how postnatal weight gain differentially affects the timing of hormonal onset of puberty could have implications for clinical management and research practices.

Although race-ethnic differences in timing of sexual maturation in US children are well documented (9), these differences have not been documented at the hormonal level around

${ }^{1}$ Hubert Department of Global Health, Rollins School of Public Health, Emory University, Atlanta, Georgia; ${ }^{2}$ Division of Pediatric Endocrinology, University of Minnesota Amplatz Children's Hospital and Medical School, Minneapolis, Minnesota; ${ }^{3}$ Department of Pediatrics, Pennsylvania State University College of Medicine, The Milton S. Hershey Medical Center, Hershey, Pennsylvania; ${ }^{4}$ Division of Gastroenterology, Hepatology and Nutrition, The Children's Hospital of Philadelphia, Philadelphia, Pennsylvania; ${ }^{5}$ Division of Epidemiology and Community Health, University of Minnesota School of Public Health, Minneapolis, Minnesota. Correspondence: O. Yaw Addo (Yaw.addo@emory.edu) 
initiation of puberty. Our study objectives are: to determine median ages at hormonal initiation of puberty based on luteinizing hormone $\mathrm{LH}$ and inhibin $\mathrm{B}(\mathrm{InB})$ and to explore the extent to which birth size, preadolescent weight gain, and raceethnicity moderate this timing in US girls.

\section{RESULTS}

The mean birthweight of the study sample was $3,256 \mathrm{~g}$ and the percentage of girls born at low birthweight (LBW) $(<2,500 \mathrm{~g})$ and those less than -1 SD BW were $6.4 \%$ and $20.8 \%$, respectively. The geometric means hormone levels for $\mathrm{LH}$ was 0.15 $\mathrm{mIU} / \mathrm{ml}$ and for InB, $9.53 \mathrm{pg} / \mathrm{ml}$. Between $23-27 \%$ of the girls had hormone values that were above the cut-points indicative of pubertal onset (Table 1). The results of adjusted multivariate models for examining the relative associations between prenatal and postnatal growth and pubertal hormones are shown in Table 2. Prenatal (BW) and postnatal weight gain were independently inversely and significantly associated with both hormone levels such that, a $1 \mathrm{SD}$ increase in birthweight and postnatal weight gain were associated with -0.24 and -4.3 $\log$ unit $\mathrm{mIU} / \mathrm{ml}$ reductions in $\mathrm{LH}$, respectively. Similarly the birthweight and postnatal weight gain were associated with -0.1 and $-0.8 \log$ unit $\mathrm{pg} / \mathrm{ml}$ changes in $\mathrm{InB}$ respectively.

The hazard ratios for exceeding the hormone cut-off indicative of pubertal onset based on anthropometry are as shown in Table 3. Birthweight was not associated with the risk of exceeding either cut-off. Postnatal relative gain was not associated with the LH cut-off, but was associated with a reduced

Table 1 Descriptive characteristics of the study sample

\begin{tabular}{|c|c|}
\hline & Girls, $N=698$ \\
\hline Descriptors & Mean (SE) \\
\hline \multicolumn{2}{|l|}{ Continuous } \\
\hline Birthweight, $g$ & $3,256(46)$ \\
\hline Age, y & $9.0(0.1)$ \\
\hline Weight, kg & $31.8(0.7)$ \\
\hline Height for-age Z score, SD & $0.14(0.1)$ \\
\hline BMI-for-age Z score, SD & $0.13(0.1)$ \\
\hline Luteinizing hormone (LH), $\mathrm{mlU} / \mathrm{ml}(\text { mean, } 95 \% \mathrm{Cl})^{\mathrm{a}}$ & $0.15(0.11-0.21)$ \\
\hline Inhibin B (InB), pg/ml (mean, 95\% Cl) & $9.53(8.41-10.80)$ \\
\hline Categorical & $\%$ \\
\hline Low birthweight $(<2,500 \mathrm{~g}, \%)$ & 6.4 \\
\hline Smaller birthweight (less than -1 SD, \%) & 20.8 \\
\hline $\begin{array}{l}\text { Birthweight less than }-1 \text { SD, overweight } \\
\text { preadolescent (\%) }\end{array}$ & 15.5 \\
\hline Overweight (>85th for BMI), \% ${ }^{\mathrm{b}}$ & 23.0 \\
\hline Obese (>95th for BMI), \% ${ }^{\mathrm{b}}$ & 10.3 \\
\hline Above LH cut-off for onset of puberty, $\%^{c}$ & 23.0 \\
\hline Above $\ln B$ cut-off for onset of puberty, $\%^{c}$ & 26.5 \\
\hline
\end{tabular}

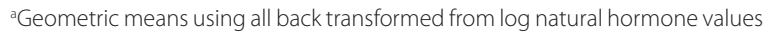

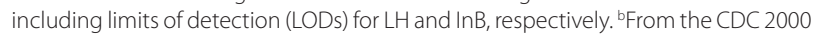
Growth Charts. From normative cut-offs ( $1.04 \mathrm{mlU} / \mathrm{ml}$ for LH and $17.89 \mathrm{pg} / \mathrm{ml}$ for InB) predictive of B2 in US children (10). All statistics adjusted for complex survey design effects. hazard for InB cut-point $(\mathrm{HR}=0.75,95 \% \mathrm{CI}$ : 62-0.91), per $1 \mathrm{SD}$ increase and for height with HR around 0.94 for both cut-offs.

The median ages at hormonal onset of puberty are shown on Figure 1 stratified by race-ethnicity and for all girls. Overall, onset occurred in a relatively tight window of $\sim 10$ mo $(9.9$ through $10.7 \mathrm{y}$ ). Pooled median age for all race-ethnicities were $10.4 \mathrm{y}$ and $10.1 \mathrm{y}$ for $\mathrm{LH}$ and $\mathrm{InB}$ respectively. Using the LH cut-points, the median age for Non-Hispanic (NH) whites (NHW) was $10.7 \mathrm{y}$ and was closer to that for Mexican American (MXAM) girls at $10.6 \mathrm{y}$, while that for their NH black peers occurred about 6 mo earlier at $10.1 \mathrm{y}$. With respect to InB, hormonal puberty initiation occurred 5-6 mo earlier in MXAM girls at $9.9 \mathrm{y}$, compared to their $\mathrm{NH}$ white and $\mathrm{NH}$ black peers.

The timing differences in hormonal initiation of puberty due to categories of birthweight, current weight BMI and postnatal weight gain are shown in Table 4. Birthweight categories were associated with the timing process for only InB but not $\mathrm{LH}$, such that those born at BW less than $-1 \mathrm{SD}$, achieved hormonal onset 12.5 mo later at $10.4 \mathrm{y}$, compared to those whose BW was greater than -1 SD 9.3 y (using InB cut-off). When median age at onset was assessed according to current BMI status, overweight girls (BMIZ greater than $+1 \mathrm{SD}$ ) achieved onset earlier $(10.3 \mathrm{y})$ in comparison to those with normal BMI (10.5 y) using LH. Puberty occurred later using InB such that median age at pubertal entry for overweight preadolescent

Table 2 Tobit models describing the associations between birthweight, postnatal relative weight gain, and hormones in peri-pubertal in US girls ages 6-11.99y

\begin{tabular}{lccccc}
\hline & \multicolumn{2}{c}{$\begin{array}{c}\text { Tobit model for } \\
\text { predicting } \mathrm{LH}, \\
\text { log } \mathrm{mlU} / \mathrm{ml}\end{array}$} & $\begin{array}{c}\text { Tobit coefficients for } \\
\text { predicting } \mathrm{InB}, \\
\text { log } \mathrm{pg} / \mathrm{ml}\end{array}$ \\
\cline { 2 - 5 } Predictors & $\begin{array}{c}\text { Coefficients } \\
\beta(\mathrm{SE})\end{array}$ & $P$ value & $\beta(\mathrm{SE})$ & $P$ value \\
\hline Birthweight (SD) & $-0.24(0.1)$ & $<0.01$ & $-0.08(0.0)$ & $<0.01$ \\
Relative weight gain (SD) & $-4.28(1.2)$ & $<0.01$ & $-0.77(0.3)$ & 0.02 \\
Height, cm & $0.15(0.01)$ & $<0.01$ & $0.05(0.0)$ & $<0.01$ \\
\hline
\end{tabular}

Models also adjusted for race-ethnicity, geographic region, health rating, and height $x$ relative wieght gain interaction. Relative weight measures the postnatal relative weight gain independent of birthweight from residual score modeling (37).

Table 3 Hazard ratios for hormonal initiation of puberty and preadolescent weight gain in US girls ages 6-11.99y

\begin{tabular}{|c|c|c|c|c|}
\hline \multirow[b]{3}{*}{ Predictors } & \multicolumn{4}{|c|}{$\begin{array}{l}\text { Hazard ratio (HR) for onset of puberty based on } \\
\text { hormone cut-points }\end{array}$} \\
\hline & \multicolumn{2}{|c|}{ Luteinizing hormone } & \multicolumn{2}{|c|}{ Inhibin B } \\
\hline & $\mathrm{HR}(95 \% \mathrm{Cl})$ & $P$ value & $\mathrm{HR}(95 \% \mathrm{Cl})$ & $P$ value \\
\hline Birthweight, SD & $1.03(0.88-1.21)$ & 0.67 & $1.12(0.98-1.28)$ & 0.05 \\
\hline $\begin{array}{l}\text { Relative weight } \\
\text { gain, (SD) }\end{array}$ & $1.04(0.88-1.23)$ & 0.62 & $0.75(0.62-0.91)$ & $<0.01$ \\
\hline $\begin{array}{l}\text { Attained height, } \\
\mathrm{cm}\end{array}$ & $0.93(0.91-0.96)$ & $<0.01$ & $0.94(0.91-0.96)$ & $<0.01$ \\
\hline
\end{tabular}


a

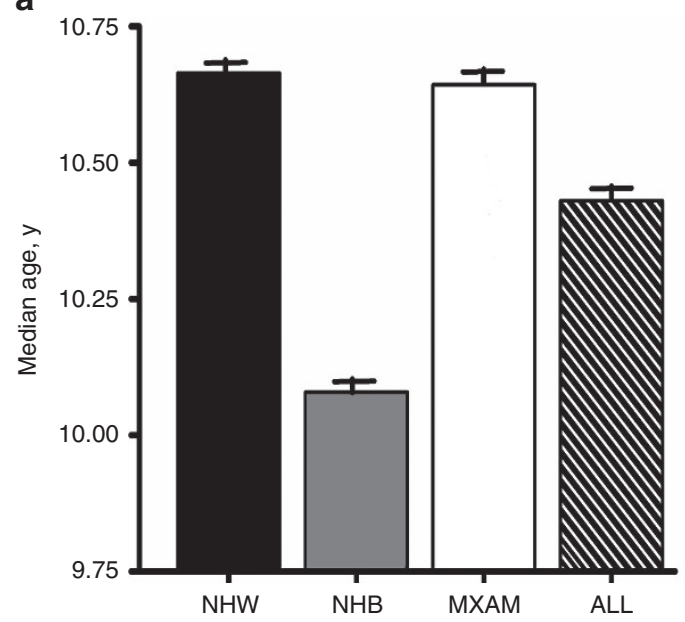

b

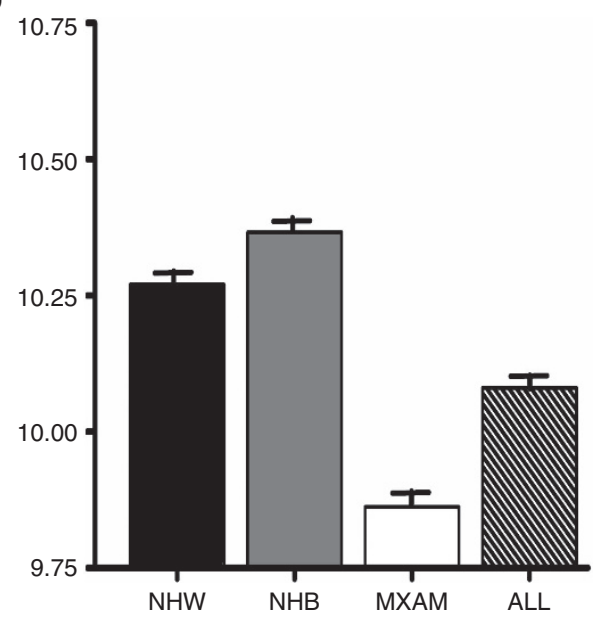

Figure 1 Estimated median age at hormonal onset of puberty based on luteinizing hormone (LH) and inhibin B1 (InB) for preadolescent US girls ages 6-11.99 y. (a) Luteinizing hormone. (b) Inhibin B. Cut-offs: (1.04 mlU/ml for LH and $17.89 \mathrm{pg} / \mathrm{ml}$ for InB). Median ages, y (95\% confidence intervals): For LH: NHW= 10.664 (10.659-10.669); NHB = 10.079 (10.072-10.086); MXAM = 10.643 (10.634-10.653); ALL = 10.430 (10.428-10.433). For InB: NHW = 10.270 (10.267-10.274); $\mathrm{NHB}=10.366$ (10.357-10.375); MXAM = 9.862 (9.857-9.867); ALL = 10.080 (10.078-10.082). Estimates for the race-ethnicity "other" $n=34$ were included in the pooled estimates only but not on their own due to the small sample size. MXAM, Mexican-American; NHB, non-Hispanic black; NHW, non-Hispanic white.

Table 4 Timing of hormonal onset of puberty based on luteinizing hormone and inhibin B in peri-pubertal US girls ages 6-11.99y

\begin{tabular}{|c|c|c|}
\hline \multirow[b]{3}{*}{ Anthropometry } & \multicolumn{2}{|c|}{ Median ages at exceeding hormonal cut-points for puberty ${ }^{a}$} \\
\hline & Luteinizing hormone & Inhibin B \\
\hline & Age, y (95\% Cls) & Age, y (95\% Cls) \\
\hline Birthweight (less than -1 SD) & $9.872(9.394-10.511)$ & $10.359(10.355-10.362)$ \\
\hline Birthweight (greater than -1 SD) & $10.323(10.000-10.738)^{b}$ & $9.319(9.316-9.322)$ \\
\hline Normal (less than \pm 1 SD BMI) & $10.460(10.457-10.463)$ & $9.910(9.908-9.912)$ \\
\hline Overweight (greater than +1 SD) & $10.301(10.295-10.308)$ & $10.506(10.502-10.511)$ \\
\hline \multicolumn{3}{|l|}{ Born small-catch-up (birth size + current BMI) } \\
\hline Birthweight less than -1 SD, normal BMI preadolescent & $10.627(10.623-10.631)$ & $10.240(10.237-10.243)$ \\
\hline Birthweight less than -1 SD but overweight preadolescent & $9.799(9.795-9.803)$ & $9.393(9.389-9.397)$ \\
\hline
\end{tabular}

girls occurred $\approx 6$ mo later compared to their normal BMI peers, at $10.5 \mathrm{y}$ and $9.9 \mathrm{y}$, respectively and opposite that of LH.

When ages of onset were determined based on composite body composition, girls born small but overweight as preadolescents had relatively earlier (9-10 mo) onset of puberty based on both $\mathrm{LH}$ and InB compared to those born small but were normal weight preadolescents. A similar pattern was observed when postnatal relative weight gain (independent of birthweight) was used. With LH, top weight gainers had a median age of $9.9 \mathrm{y}$ and about 11 mo earlier compared to normal gainers (10.8 y). Also, for this normal group, using InB, median onset occurred at $10.0 \mathrm{y}$ and 6 mo earlier than that of the top gainers at $10.5 \mathrm{y}$.

\section{DISCUSSION}

Using a nationally representative sample of US girls ages 6-11.99 y, we find that fetal growth denoted by birthweight, and preadolescent relative weight gain are both independently and inversely associated with $\mathrm{LH}$ and InB levels in peri-pubertal US girls. From probit models, the median ages at hormonal onset of puberty were $10.4 \mathrm{y}$ based on $\mathrm{LH}$, and $10.1 \mathrm{y}$ for $\operatorname{InB}$ cut-offs, and both median ages were significantly associated with race-ethnicity, birthweight and postnatal weight gain. This study attempts to address one of the key recommendations by an expert panel concerning the importance of physiologic markers of puberty on timing for ascertaining later phenotypic outcomes (2). This has been challenging because 
hormonal data on puberty in a national sample of children have not been available until this NHANES III study. Together with the recently published normative growth curves for luteinizing hormone and inhibin B in peri-pubertal girls (10), the hormonal onset puberty ages derived in this study will inform research and practice on some of the physiological mediators of puberty in US girls.

While some assert that breast bud exam by a pediatrician can reliably determine pubertal staging, it can be inaccurate in obese girls. It also does not predict the onset of puberty based upon hypothalamic-pituitary secretion as the presence of breast development at any age does not necessarily mean puberty has begun unless it is followed by progression or verified by hormone levels. It has been well documented that both LH and inhibin B levels increase during puberty, particularly early puberty. Mean circulating levels of $\mathrm{LH}$, together with the other gonadotropin, follicle-stimulating hormone (FSH), increase beginning before physical changes of puberty begin (11). Inhibin B levels begin to rise concomitant with early gonadal growth $(10,12,13)$. These, as well as other reproductive system hormones, are essential to attain and maintain sexual maturity. Evaluation of LH in the analyses of this report was chosen because of its essential role and because the increase in circulating levels is more robust than FSH.

Inhibin B was chosen because it is a marker of ovarian function that increases with sexual maturation and regulates FSH production. It is also a gonadal function marker in girls with abnormalities of the hypothalamic-pituitary axis (14). While it can be argued that a single measurement of LH is insufficient to indicate the onset of puberty, there is evidence that this is adequate if the level is within the pubertal range, above the overlap of prepubertal and pubertal levels (15). FSH levels however do not rise dramatically with pubertal onset in girls. Estradiol levels generally fluctuate so much that it is unusual among pubertal girls when a level falls within the pubertal range. While inhibin $B$ has not been as extensively studied, the rise of levels at the onset of puberty was felt to merit further investigation. Since serum was not available to assay other hormones, the analyses presented here further define InB levels in relation to the onset of puberty.

This study also advances existing research as we elucidate relations between prenatal and postnatal body growth and hormone data in a US national sample. Even though many past studies have examined associations between body composition and puberty in girls (16), a majority have used menarche as the measure of puberty, even though it is a downstream marker of the maturational process. We overcome this and other methodological shortcomings of prior research as we assess the relative contribution of each growth period on reproductive hormones, and explored how body composition relates to risk of exceeding hormone cut-offs indicative of puberty.

\section{Pre- and Postnatal Body Composition and Pubertal Hormones}

We found no significant simple correlations between birthweight and either hormone, but postnatal weight gain was positively correlated with $\mathrm{LH}$ but not $\operatorname{InB}$ (data not shown).
In Tobit models accounted for hormone assay LODs, however, postnatal weight gain was found to be more strongly and inversely related to both pubertal hormone levels (LH and InB) relative to birthweight. This could imply that, while onset of puberty may have prenatal origins, postnatal growth, weight gain probably, plays a much greater role in US girls. Girls who were born "smaller" (BW less than -1 SD) but were overweight as preadolescents initiated hormonal (as defined by LH concentrations) puberty 9-10 mo earlier than their peers born small but with healthy catch-up (normal BMI). These results suggest that adverse prenatal circumstances like intra-uterine growth restriction (IUGR) and or preterm birth may have implications on pubertal maturation later in life, especially in settings with high childhood obesity. It is also consistent with findings in SGA infants that undergo catch-up early who are reported to have problems with insulin resistance, premature adrenarche, and PCOS (17) later in life. This further highlights the possible importance of obesity reflecting nutrition in addition to, perhaps environmental endocrine disruptors (18).

Height (attained size) was associated with a slightly reduced hazard of pubertal onset (via LH and InB cut-points) in models that were concurrently adjusted for postnatal relative weight gain. As height could also be capturing lean mass at this stage in life, these results suggests that observed associations may be driven by postnatal ponderosity (lean and fat masses). A fat mass relationship could also suggest a role of leptin or other fat-related signal regulating central puberty, but not regulating gonadal function. This potential mechanism is consistent with past work that found leptin to be strongly correlated with fatness (19) and has also been suggested for initiating changes in pubertal hormones such as LH, FSH and estradiol in girls (20-22).

Preadolescent relative weight gain was positively (but nonsignificantly) associated with later onset for LH-defined but with earlier and significant onset based on InB-defined pubertal cut-offs. These opposing results could highlight possible implications of body composition in the polycystic ovarian syndrome (PCOS) (23) as was observed a previous study in which BMI inversely correlated with inhibin B in women with PCOS (24). This could suggest that, the relation between obesity and reproductive dysfunction may operate by negatively impacting hormonal markers of gonadal maturation, even in preadolescence.

Several other potential mechanisms, like differences in metabolism, hormone clearance and sequestration in girls that grow with high adiposity could partly explain the discordant associations (25), which were also observed in past studies $(26,27)$ involving women. Furthermore, it is also probable that LH may operate together with high adiposity to attenuate observed associations for InB-defined puberty in girls. When this potential interpretation was examined in our study data, we found that, while InB was positively associated with $\mathrm{LH}$, it was also negatively and more strongly associated with weight gain (data not shown). This might have resulted in a combined negative effect (of both LH and adiposity) on InB and could in part, explain these discordant findings. An additional 
interpretation could be via the suppressive effect of high adiposity on hypothalamic-pituitary gonal function (28) coupled with hyperandrogenemia (29), independent of its known paradoxic role of advancing puberty (28). Further research in children is needed to fully elucidate this complex interplay of hormones, nutrition and adiposity associated with physical growth and maturation and adverse health outcomes through young adulthood.

\section{Ages at Hormonal Onset of Puberty and Body Composition}

We calculated probit median ages at hormonal onset of puberty based on hormone cut-points predictive of physical signs of puberty, B2 (10). The overall median age at of onset of hormonal puberty ranged from 10.1 to $10.4 \mathrm{y}$, which is consistent with approximate ages at "take-off" often used to describe skeletal growth in the United States (30) and Europe (31), and estimated by a variety of growth models and attainment of physical characteristics like breast stage II $(32,33)$ in girls. Only $<5 \%$ of our sample at breast stage I had LH and InB levels above either cut-points indicative of pubertal onset, with only $1 \%(n=7)$ above both cut-points. Together, these results suggest that the estimated ages at hormonal onset of puberty captures auxological milestones that typify normal growth.

The median ages at hormonal onset based on LH were 10.7, 10.1 and $10.6 \mathrm{y}$ for NHW, NHB, and MXAM girls, respectively. The race-ethnic ranking of the timing (NHB $<<\mathrm{MXAM}<<$ NHW, "early" to "late" onset) observed in this study is also consistent with that from previous research on national age at menarche (NHB: 12.06, MXAM: 12.25; NHW: 12.55) (9). Although initiation of hormonal (LH) onset of puberty occurred earlier in NHB, it took them longest, $\sim 2$ y (1.98) to reach menarche, followed by NHW with $1.89 \mathrm{y}$ and the shortest in MXAM girls at $1.61 \mathrm{y}$. Using LH levels as a potential marker of the pubertal onset and menarche as the end of puberty, our findings show that MXAM girls progress through the maturation process the quickest, allowing the least time for pubertal growth. This could be due to factors like genetics, nutrition and obesity. A recent systematic review found that excess body weight in infancy advances several pubertal indicators including age at menarche (31). The present analyses corroborates this finding at the hormonal level, as we found that top weight gainers (and overweight) girls initiated hormonal (by LH) puberty 10.8 mo earlier than normal weight gain girls.

\section{Strengths and Limitations}

The estimation of a nationally representative normative age at hormonal onset of puberty based on $\mathrm{LH}$ and InB, make our findings largely generalizable to children of all the major race-ethnicities in the United States. The use of residual score modeling for relative importance assessments that controls for tendency for growth tracking, and the use of Tobit models capable of addressing hormone assay's with LODs are some strengths of this study. This research also has limitations. The research findings are based on analyses of frozen sera data collected from 1988 to 1994 and may be subject to secular changes in body composition that have occurred in the United
States since then. It does seem unlikely that the relationships among variables should change even if the exact hormone and body composition levels may have changed.

We are unable to examine how hormones such as growth hormone $(\mathrm{GH})$, insulin-like growth factor 1 (IGF-1), estradiol, or anti-mullerian hormone, among several others, are related to body composition around onset of puberty. Birthweights were obtained via maternal recall and may be subject to recall biases. LBW prevalence was only $6.4 \%$ and as a result of such a small sample we could not reliably examine its relations to puberty. Since gestational age data was unavailable, we could not examine how adverse fetal growth circumstances like SGA, and preterm births mediate pubertal timing.

\section{Conclusions}

This research provides national normative median ages at hormonal onset of puberty for practice and research, based on LH and InB. Postnatal weight gain is more strongly related with these hormone levels than prenatal growth and higher postnatal weight gain lowers the age of hormonal onset of puberty as defined by LH concentrations. Preventing obesity in childhood could also avert the earlier initiation of the maturation process at the hormonal level, and may allow sufficient time for achievement of full growth potential.

\section{METHODS}

We analyzed anthropometric and hormone data of 698 US peripubertal girls ages 6-11.99 y who participated in the Third National Health and Nutrition Examination Survey (NHANES III) from 1988 to 1994 , a nationally representative survey of noninstitutionalized Americans (34). Anthropometric measures were collected following standard protocols and included birthweight (BW); current weight and height as well as body mass index (BMI estimated as Weight kg/ (Height, $\mathrm{m}^{2}$ ). Other demographic variables such as race-ethnicity, poverty income ratio, health rating, and geographic region were also available (34). We applied and were provided frozen serum to be limited to the measurement of $\mathrm{LH}$ and inhibin $\mathrm{B}$, as there was inadequate sample volume for the requirement for estradiol using a sensitive assay. The Research Ethics Review Board of the National Center for Health Statistics, US Centers for Disease Control and Prevention approved the NHANES study. Informed consent was obtained from each participant before the survey, as well as from parents/guardians of minors.

\section{Laboratory Analyses}

Hormonal assays (InB and $\mathrm{LH}$ ) were conducted on nonfasting serum samples that were collected from 1988 to 1994 and stored at $-70{ }^{\circ} \mathrm{C}$. Inhibin B and LH were measured using Gen I DSL-10841000 ACTIVE Inhibin B ELISA (Diagnostic Systems Laboratories, Webster, TX) and LH ELISA kit (Bio-Quant BQ049F; Bio-Quant, San Diego, CA) respectively. Hormone limits of detection (LODs) were set $a$ priori by the assay manufacturers. The NHANES blood sampling were divided in three sessions: morning (8:00 am to $12: 59 \mathrm{pm}, 38.7 \%$ of our study sample), afternoon (1:00 pm to 5:00 pm, 35.1\%), and evening ( $>5: 00 \mathrm{pm}, 26.1 \%)$. Since LH levels vary considerably throughout the day in early puberty, analysis of variance tests were carried out to see if the session of blood draw might have influenced hormone distributions. No significant differences were observed in LH or InB irrespective of the blood draw session $\left(\mathrm{LH} \mathrm{P}_{\text {trend }}=0.97\right.$; InB $\mathrm{P}_{\text {trend }}=$ 0.43; data not shown) and thus, pooled analysis was conducted.

\section{Statistical Methods}

Descriptive statistics are presented as means (SE) or as geometric means (95\% CIs) where appropriate. InB and $\mathrm{LH}$ were used as study outcomes while BW, relative weight gain were used as study 
exposures. Overweight status was defined as BMI greater than $+1 \mathrm{SD}$ score and "small birth size" as BW less than -1 SD score (35). We also created a categorical composite variable for children who had small birth size but had healthy "catch-up" (normal BMI <85th percentile, vs. overweight) in preadolescence. Complex survey design effects were applied (36), where appropriate in order to generate weighted, nationally representative results. Due of the nature the NHANES of sampling design and case weighting for nonresponse, routine statistics are often generated with standard error as better measure of precision around estimates rather than standard deviations.

\section{Relative Weight Gain}

We partitioned growth into prenatal and postnatal components using residual score modeling (37) by estimating relative postnatal weight gain independent of birthweight. This derived variable could be regarded as a standardized measure of relative weight gain velocity. Accordingly, participants with postnatal weight gain greater than +1 SD could be termed "fast or top gainers." Since both BW and postnatal relative weight gain are uncorrelated and scaled into SD units, they both assess the distinct contributions of the two periods to the study outcomes (LH and InB) in a regression model.

\section{Age at Hormonal Onset of Puberty and Body Weight}

The ages at hormonal onset of puberty were calculated using probit models. The hormone cut-points $1.04 \mathrm{mIU} / \mathrm{ml}$ for $\mathrm{LH}$ and $17.89 \mathrm{pg} /$ $\mathrm{ml}$ for InB previously derived to be predictive of breast stage 2(B2) in US children (10) were used as binary outcome variables. The probit age estimation used in this research is a "status quo" method (38) and applicable to cross-sectional hormone data. The ages at onset were calculated with their 95\% fiducial limits (FL). Following Fieller's Theorem (39), we used 95\% confidence intervals (CIs) as the fiducial analogue (40) for hypothesis testing throughout this report.

\section{Regression Analyses}

Tobit and Cox proportional hazard models were used to study associations. We examined the relative contributions of birthweight (prenatal) and postnatal weight gain on continuous pubertal hormones using Tobit models, which are capable of handling hormone distribution left truncation due to assay LODs. In order to also manage the time effects on a life-span event like pubertal onset, complex survey Cox hazard models were used to examine the relations between anthropometry and the hazard ratios (HR, 95\% CIs) for exceeding the hormonal puberty cut-points. Poverty income ratio, race-ethnicity, size (height), age, census region, and general state of health were controlled for as potential confounders or covariates based on theory.

Statistical analyses were conducted in $\mathrm{R}$ ( $\mathrm{R}$ foundation for Statistical Computing, Vienna, Austria) and SAS 9.3(SAS Institute, Cary, NC). Statistical significance was set at a two-sided $\alpha$ of $5 \%$.

Disclosure: The authors declare no conflict of interest.

\section{REFERENCES}

1. Tinggaard J, Mieritz MG, Sørensen K, et al. The physiology and timing of male puberty. Curr Opin Endocrinol Diabetes Obes 2012;19:197-203.

2. Euling SY, Herman-Giddens ME, Lee PA, et al. Examination of US pubertytiming data from 1940 to 1994 for secular trends: panel findings. Pediatrics 2008;121:Suppl 3:S172-91.

3. Parent AS, Teilmann G, Juul A, Skakkebaek NE, Toppari J, Bourguignon JP. The timing of normal puberty and the age limits of sexual precocity: variations around the world, secular trends, and changes after migration. Endocr Rev 2003;24:668-93.

4. Gollenberg AL, Hediger ML, Lee PA, Himes JH, Louis GM. Association between lead and cadmium and reproductive hormones in peripubertal U.S. girls. Environ Health Perspect 2010;118:1782-7.

5. Sloboda DM, Hart R, Doherty DA, Pennell CE, Hickey M. Age at menarche: Influences of prenatal and postnatal growth. J Clin Endocrinol Metab 2007;92:46-50.

6. Tam CS, de Zegher F, Garnett SP, Baur LA, Cowell CT. Opposing influences of prenatal and postnatal growth on the timing of menarche. J Clin Endocrinol Metab 2006;91:4369-73.

7. DiVall SA, Radovick S. Pubertal development and menarche. Ann N Y Acad Sci 2008;1135:19-28.
8. Aksglaede L, Olsen LW, Sørensen TI, Juul A. Forty years trends in timing of pubertal growth spurt in 157,000 Danish school children. PLoS ONE 2008;3:e2728.

9. Chumlea WC, Schubert CM, Roche AF, et al. Age at menarche and racial comparisons in US girls. Pediatrics 2003;111:110-3.

10. Sims EK, Addo OY, Gollenberg AL, Himes JH, Hediger ML, Lee PA. Inhibin $\mathrm{B}$ and luteinizing hormone levels in girls aged 6-11 years from NHANES III, 1988-1994. Clin Endocrinol (Oxf) 2012;77:555-63.

11. Lee P. Physiology of puberty. In: Becker K, ed. Principles and Practice of Endocrinology and Metabolism. Philadelphia: JB Lippincott, 2001:885-93.

12. Sehested A, Juul AA, Andersson AM, et al. Serum inhibin A and inhibin B in healthy prepubertal, pubertal, and adolescent girls and adult women: relation to age, stage of puberty, menstrual cycle, follicle-stimulating hormone, luteinizing hormone, and estradiol levels. J Clin Endocrinol Metab 2000;85:1634-40.

13. Lahlou N, Roger M. Inhibin B in pubertal development and pubertal disorders. Semin Reprod Med 2004;22:165-75.

14. Cuny A, Trivin C, Brailly-Tabard S, et al. Inhibin B and anti-Müllerian hormone as markers of gonadal function after treatment for medulloblastoma or posterior fossa ependymoma during childhood. J Pediatr 2011;158:1016-1022.e1.

15. Houk CP, Kunselman AR, Lee PA. Adequacy of a single unstimulated luteinizing hormone level to diagnose central precocious puberty in girls. Pediatrics 2009;123:e1059-63.

16. Ong KK, Emmett P, Northstone K, et al. Infancy weight gain predicts childhood body fat and age at menarche in girls. J Clin Endocrinol Metab 2009;94:1527-32.

17. Longo S, Bollani L, Decembrino L, Di Comite A, Angelini M, Stronati M. Short-term and long-term sequelae in intrauterine growth retardation (IUGR). J Matern Fetal Neonatal Med 2013;26:222-5.

18. Blanck HM, Marcus M, Tolbert PE, et al. Age at menarche and tanner stage in girls exposed in utero and postnatally to polybrominated biphenyl. Epidemiology 2000;11:641-7.

19. Demerath EW, Towne B, Wisemandle W, Blangero J, Chumlea WC, Siervogel RM. Serum leptin concentration, body composition, and gonadal hormones during puberty. Int J Obes Relat Metab Disord 1999;23:678-85.

20. Matkovic V, Ilich JZ, Badenhop NE, et al. Gain in body fat is inversely related to the nocturnal rise in serum leptin level in young females. J Clin Endocrinol Metab 1997;82:1368-72.

21. Xi H, Zhang L, Guo Z, Zhao L. Serum leptin concentration and its effect on puberty in Naqu Tibetan adolescents. J Physiol Anthropol 2011;30:111-7.

22. Rutters F, Nieuwenhuizen AG, Verhoef SP, Lemmens SG, Vogels N, Westerterp-Plantenga MS. The relationship between leptin, gonadotropic hormones, and body composition during puberty in a Dutch children cohort. Eur J Endocrinol 2009;160:973-8.

23. Dunger DB, Ahmed ML, Ong KK. Effects of obesity on growth and puberty. Best Pract Res Clin Endocrinol Metab 2005;19:375-90.

24. Welt CK, Taylor AE, Martin KA, Hall JE. Serum inhibin B in polycystic ovary syndrome: regulation by insulin and luteinizing hormone. J Clin Endocrinol Metab 2002;87:5559-65.

25. Su HI, Sammel MD, Freeman EW, Lin H, DeBlasis T, Gracia CR. Body size affects measures of ovarian reserve in late reproductive age women. Menopause 2008;15:857-61.

26. Cortet-Rudelli C, Pigny P, Decanter C, et al. Obesity and serum luteinizing hormone level have an independent and opposite effect on the serum inhibin B level in patients with polycystic ovary syndrome. Fertil Steril 2002;77:281-7.

27. De Pergola G, Maldera S, Tartagni M, Pannacciulli N, Loverro G, Giorgino R. Inhibitory effect of obesity on gonadotropin, estradiol, and inhibin B levels in fertile women. Obesity (Silver Spring) 2006;14:1954-60.

28. Rosenfield RL, Bordini B. Evidence that obesity and androgens have independent and opposing effects on gonadotropin production from puberty to maturity. Brain Res 2010;1364:186-97.

29. McCartney CR, Blank SK, Prendergast KA, et al. Obesity and sex steroid changes across puberty: evidence for marked hyperandrogenemia in preand early pubertal obese girls. J Clin Endocrinol Metab 2007;92:430-6.

30. Berkey CS, Dockery DW, Wang X, Wypij D, Ferris B Jr. Longitudinal height velocity standards for U.S. adolescents. Stat Med 1993;12:403-14. 


\section{Articles | Addo et al.}

31. Butler GE, McKie M, Ratcliffe SG. The cyclical nature of prepubertal growth. Ann Hum Biol 1990;17:177-98.

32. Lee PA. Normal ages of pubertal events among American males and females. J Adolesc Health Care 1980;1:26-9.

33. Foster TA, Voors AW, Webber LS, Frerichs RR, Berenson GS. Anthropometric and maturation measurements of children, ages 5 to 14 years, in a biracial community-the Bogalusa Heart Study. Am J Clin Nutr 1977;30:582-91.

34. Centers for Disease Control. The National Health and Nutrition Examination Survey, 2014. (http://www.cdc.gov/nchs/nhanes.htm).

35. Kuczmarski RJ, Ogden CL, Grummer-Strawn LM, et al. CDC growth charts: United States. Adv Data 2000;314:1-27.
36. Korn EL, Graubard BI. Epidemiologic studies utilizing surveys: accounting for the sampling design. Am J Public Health 1991;81:1166-73.

37. Keijzer-Veen MG, Euser AM, van Montfoort N, Dekker FW, Vandenbroucke JP, Van Houwelingen HC. A regression model with unexplained residuals was preferred in the analysis of the fetal origins of adult diseases hypothesis. J Clin Epidemiol 2005;58:1320-4.

38. Macmahon B. Age at Menarche, United States. Series 11: 133NCHS DHEW Publ. No. (HRA) 74-1615. Washington, DC: National Center for Health Statistics, Vital and Health Statistics, 1973.

39. Fieller E. Some problems in interval estimation. J R Stat Soc Series B Stat Methodol 1954;16:175-85.

40. Wang Y. Fiducial intervals: what are they? Am Stat 2000;54:105-11. 